\title{
Liposarcoma of the Spermatic Cord: An Infrequent Pathology
}

\author{
Marcelo Di Gregorio ${ }^{a} \quad$ Lionel D'Hondt ${ }^{b}$ Francis Lorge ${ }^{a}$ \\ Marie-Cécile Nollevaux ${ }^{c}$ \\ aUrology Department, CHU UCL, Namur, Belgium; ${ }^{b}$ Oncology Department, CHU UCL, \\ Namur, Belgium; 'Pathology Department, CHU UCL, Namur, Belgium
}

\section{Keywords}

Liposarcoma $\cdot$ Spermatic cord · Testicular neoplasms · Orchiectomy

\begin{abstract}
Objective: To describe a rare pathology, the liposarcoma of the spermatic cord (LSC), and discuss its diagnosis and treatment. Materials and Methods: We report a case of welldifferentiated LSC in a 61-year-old man. The main complaint was painless enlargement in the right inguinoscrotal area that appeared 6 months prior to presentation. Ultrasonography showed a heterogeneous and hyperechogenic mass of the right spermatic cord that was highly suspicious of malignancy. Computed tomography confirmed a heterogeneous supratesticular mass. The mass was surgically removed. Results: Pathological examination showed a well-differentiated LSC. The patient did not receive any additional treatment. The follow-up did not present complications or further symptoms and, at present, the patient is in complete remission. Conclusion: LCSs are a very rare entity. The diagnosis is difficult and often mistaken with common scrotal swelling associated with a hernia, hydrocele, or other tumour. Treatment should include complete surgical excision, usually by radical inguinal orchiectomy. A long follow-up period is necessary.




\section{Case Reports in Oncology}

Di Gregorio et al.: Liposarcoma of the Spermatic Cord: An Infrequent Pathology

\section{Introduction}

Malignant lesions of the spermatic cord are rare, with about 200 cases reported in the literature [1-3]. Liposarcomas are malignant neoplasms of adipose tissue that arise from mesenchymal cells. In almost $70 \%$ of cases, liposarcomas are located in the extremities or in the retroperitoneum. Liposarcoma of the spermatic cord (LSC) is a rare entity, representing approximately $7 \%$ of paratesticular sarcomas [4]. The spermatic cord is the most commonly involved urologic site, accounting for approximately $30 \%$ of all genitourinary sarcomas $[1$, 5].

Diagnosis is sometimes difficult and unexpected, and the differential diagnosis can be inguinal hernia, funicular cyst, or lipoma of the cord $[6,7]$. The recommended treatment is surgery with wide local excision, which in most cases, means a radical high orchiectomy performed as close as possible to the deep ring of the inguinal canal. Adjuvant radiotherapy is proposed in cases with positive margins or when there is local recurrence or poor prognostic factors.

However, these cancers are known for their local recurrence, so long-term follow-up of $10-20$ years is mandatory $[8,9]$.

\section{Clinical Presentation}

A 61-year-old man noticed a progressive painless swelling in the right inguinoscrotal region that was present for over 6 months. He noticed a more rapid growth in the 6 weeks prior to consultation, with the swelling growing to $8 \times 5 \mathrm{~cm}$ at presentation. There was no cough impulse for the swelling. Palpation revealed a firm, smooth-surfaced tender swelling. The trans-illumination test was negative, and there was no associated inguinal lymphadenopathy.

Ultrasonography revealed a heterogeneous hyperechogenic mass with mixed echogenicity involving the right spermatic cord. This pattern was highly suspicious of malignancy (Fig. $1 \mathrm{a}, \mathrm{b})$. The chest-abdominopelvic computed tomography (CT) scan showed a heterogeneous right supratesticular mass, essentially suety tissue, that measured $84 \times 78 \times 72 \mathrm{~mm}$ (Fig. 1c, d), without metastatic lesion.

Surgery was performed using an inguinal approach. The spermatic cord was dissected and removed, and it showed a mass that was $14 \times 8 \mathrm{~cm}$ in size. High right radical inguinal orchiectomy was performed. The mass involved the entire circumference of the cord and could not be separated from the cord (Fig. 2a). Macroscopic examination of the testis showed that it appeared to be normal (Fig. 2b). The mass had a "bunch of grapes" appearance and consisted of several masses of various sizes that surrounded the spermatic cord (Fig. 2c). Histopathological examination revealed a well-differentiated liposarcoma of the cord with positive margins upon resection (Fig. 2d). The patient underwent a second surgery to resect the remaining endoperitoneal portion of the spermatic cord 5 weeks later. The subsequent histopathological examination showed nonresidual neoplastic tissue.

The patient has not received any adjuvant treatment, and the 2-year follow-up revealed no locoregional recurrence or tumour progression without adjuvant therapy. 


\section{Discussion}

In 1845, Lesauvage reported the first case of sarcoma of the cord [4]. Lipomatous tissue plays a minor role in seminal cord malignancies, representing about $5-7 \%$ of all spermatic cord sarcomas [3].

Liposarcoma is a disease that mostly affects older individuals, and it usually presents as a slow-growing inguinal or inguinoscrotal mass. The average patient age at presentation is 55 years (range, 16.5-85) [10].

Staging of these sarcomas is based on histological grade and on the presence of metastasis. The Word Health Organisation classification of soft tissue tumours recognizes 5 categories of liposarcomas of increasing malignancy: (a) well differentiated, which includes the adipocytic, inflammatory, and sclerosing subtypes; (b) dedifferentiated; (c) myxoid; (d) round cell, and (e) pleomorphic [4, 7].

Liposarcoma of the cord needs to be differentiated from other paratesticular masses like incarcerated hernias, hydroceles, benign tumours (lipoma, leiomyoma, haemangioma), and malignant tumours (rhabdomyosarcoma and melanotic neuroectodermal tumour of infancy). It generally remains asymptomatic for years, but it may have potentially life-threatening sequelae $[8,9,11]$.

Currently, nonspecific diagnostic procedures are recommended for evaluating a scrotal mass. Ultrasonography provides little information on paratesticular sarcomas. There are nonpathognomonic features defined in the literature that help differentiate between benign and malignant masses $[8,12]$. Pleomorphic-type liposarcoma presents as a heterogeneous mass that shows many hyper- and hypoechoic nodules on ultrasonography, and it can be distinguished easily from lipoma.

The use of CT scans for liposarcomas is not widely described, but this seems promising. Nonetheless, there are no pathognomonic features for differentiating between benign and malignant masses [12]. MRI provides good information for the local situation, but it cannot provide complete characterization of the mass. Fluorodeoxyglucose (FDG)-PET scanning may be useful in recurrent cases, but its routine use is not indicated.

Histologically, almost all of these cancers are well-differentiated. Low-grade malignancies have no or a minimal tendency to metastasize, but they may be locally invasive $[1,7,8]$.

The recommended treatment for liposarcoma is surgery and specifically radical high orchiectomy. However, the anatomical features of the inguinal region sometimes make it difficult to perform this procedure, and negative resection margins are sometimes close to the tumours. A second resection is advised if the margins are positive, to avoid misleading conclusions.

In the present case, the margins were positive and a second-look surgery was performed to resect the intraperitoneal spermatic cord segment. This second resection achieved negative margin status. At the uro-oncology tumour meeting, it was decided that follow-up was appropriate for this patient. After 30 months of follow-up, the patient remains in complete remission.

Recurrence is managed by surgery or radiotherapy and, rarely, by chemotherapy. Longterm follow-up is mandatory, since recurrences after 18 years have been reported [2, 9].

Liposarcomas are the most radiosensitive of all sarcomas, and in some cases, remission has been achieved with radiotherapy alone $[8,13]$. Two prospective randomized trials involving soft tissue sarcoma established that the addition of radiation to surgery substantially reduces the likelihood of local recurrence [13]. 
However, the previously reported liposarcoma series are not sufficiently large to clearly demonstrate the need for additional treatment, due to the radiosensitivity. Radiation therapy is recommended in addition to surgery for high-grade tumours, lymphatic invasion, inadequate margins, or relapses.

In the present case, the tumour was a well-differentiated liposarcoma of the cord, and the resection showed positive margins. The patient underwent a second surgical procedure to remove the remaining endoperitoneal portion of the spermatic cord. The histopathological examination showed nonresidual neoplastic tissue, so no additional treatment was proposed at the time. Radiotherapy can be delayed or postponed until relapse if the patient is followed up closely.

Liposarcomas have a good prognosis because of their low degree of malignancy and because relapses are usually local. There is a high 5-year survival rate [14].

\section{Conclusion}

LSCs are a very rare entity. Preoperatively, the diagnosis is difficult. Treatment should include complete surgical excision, usually by radical inguinal orchiectomy. If the margin status is doubtful, a second look and excision of the intraperitoneal portion of the spermatic cord is mandatory. Adjuvant radiation treatment can be discussed, especially for welldifferentiated tumours. Distant disease has not been reported, but local relapse is common and may occur even several years after primary therapy. A long follow-up period is necessary.

\section{Acknowledgements}

The authors acknowledge Christian Deneffe for picture edition and Edith Botek for reading support.

\section{Statement of Ethics}

Written informed consent was obtained from the patient for publication of this case report and any accompanying images.

\section{Disclosure Statement}

The authors have no conflicts of interest to declare.

\section{Author Contributions}

M.D.G. conceived, coordinated, and designed the study. F.L. participated in its design and helped to draft the manuscript. L.D. helped in coordination and draft corrections. M.-C.N. participated in the anatomo-pathological figures and corresponding drafts. All authors read and approved the final manuscript. 


\section{References}

1 Schwartz SL, Swierzewski SJ, Sondak VK, Grossmann HB: Liposarcoma of the spermatic cord: report of 6 cases and review of the literature. J Urol 1995;153:154-157.

2 Fitgerald S, Maclennan GT: Paratesticular liposarcoma. J Urol 2009;181:331-332.

- 3 Tandon M, Khandelwal R, Jain, S, Narayan N, Kumar Y, Saxena S: Liposarcoma of the spermatic cord: a diagnostic dilemma. JRSM Short Reports 2010;1:49.

4 Hinman F, Gibson TE: Tumors of the epididymis, spermatic cord and testicular tunics: a review of the literature and report of three new cases. Arch Surg 1924;8:100.

-5 Demirci U, Buyukberber S, Cakir A, Ozturk B, Akyurek N, Unver B, Coskun U: Synchronous testicular liposarcoma and prostate adenocarcinoma: a case report. Cases J 2010;3:1.

-6 Li F, Tian R, Yin C, Dai X, Wang H, Xu N, Guo K: Liposarcoma of the spermatic cord mimicking a left inguinal hernia: a case report and literature review. World J Surg Oncol 2013;11:1.

7 Rodriguez D, Olumi AF: Management of spermatic cord tumors: a rare urologic malignancy. Ther Adv Urol 2012;4:325-334.

-8 Coleman J, Brennan MF, Alektiar K, Russo P: Adult spermatic cord sarcomas: management and results. Ann Surg Oncol 2003;10:669-675.

-9 Fagundes MA, Zietman AL, Althausen AF, Coen J, Shipley WU: The management of spermatic cord sarcoma. Cancer 1996;77:1873-1876.

10 Yoshino T, Yoneda K, Shirane T: First report of liposarcoma of spermatic cord after radical prostatectomy for prostate cancer. Anticancer Res 2009;29:677-680.

11 Ballot MT, Zagars GK, Pisters PW, et al: Spermatic cord sarcomas: outcome, patterns of failure and management. J Urol 2001;166:1306-1310.

12 Cardenosa G, Papinicolaou W. Fung CY, et al: Spermatic cord sarcomas: sonographic and CT features. Urol Radiol 1990;12:136-137.

13 Yang JC, Chang AE, Baker AR, et al: Randomized prospective study of the benefit of adjuvant radiation therapy in the treatment of soft tissue sarcomas of the extremity. J Clin Oncol 1998;16:197-203.

$\checkmark 14$ Lee HN, Kim MS: Liposarcoma of the testis and spermatic cord. Open J Urol 2013;3:68-70. 


\section{Case Reports in Oncology}

\begin{tabular}{l|l}
\hline Case Rep Oncol 2017;10:136-142 \\
\hline DOI: 10.1159/000455900 & $\begin{array}{l}\text { @ 2 2017 The Author(s). Published by S. Karger AG, Basel } \\
\text { www.karger.com/cro }\end{array}$ \\
\hline
\end{tabular}
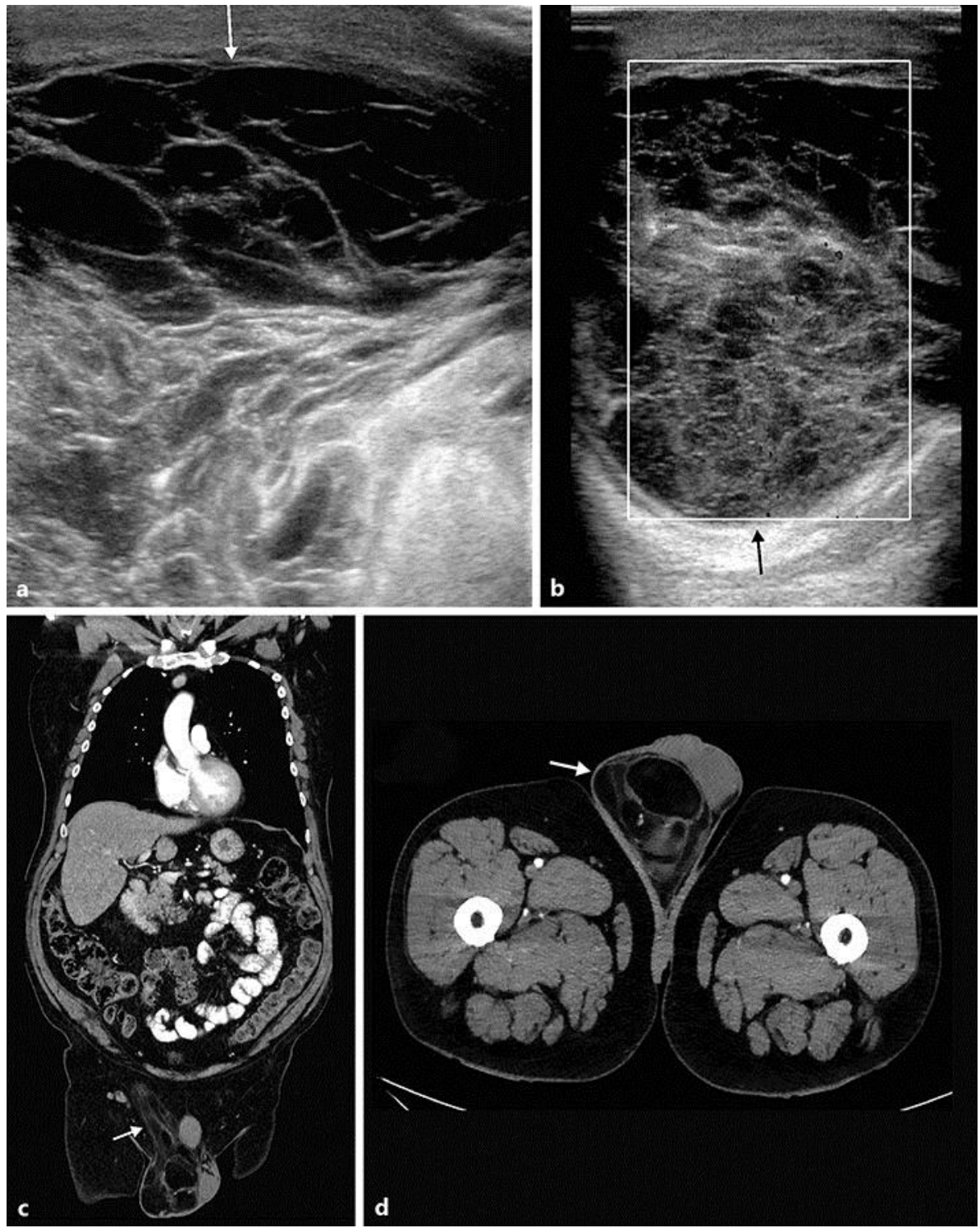

Fig. 1. Ultrasonography revealed a heterogeneous and hyperechogenic mass with mixed echogenicity involving the right spermatic cord. This is highly suspicious of malignancy. a Inguinal area. b Scrotal area. The chest-abdominal CT scan showed a heterogeneous right supratesticular mass, essentially suety tissue, measuring $84 \times 78 \times 72 \mathrm{~mm}$, without metastatic lesions. c Inguinal area. $\mathbf{d}$ Scrotal area. 


\section{Case Reports in Oncology}

\begin{tabular}{l|l}
\hline Case Rep Oncol 2017;10:136-142 \\
\hline DOI: $10.1159 / 000455900$ & $\begin{array}{l}\text { C 2017 The Author(s). Published by S. Karger AG, Basel } \\
\text { www.karger.com/cro }\end{array}$ \\
\hline
\end{tabular}
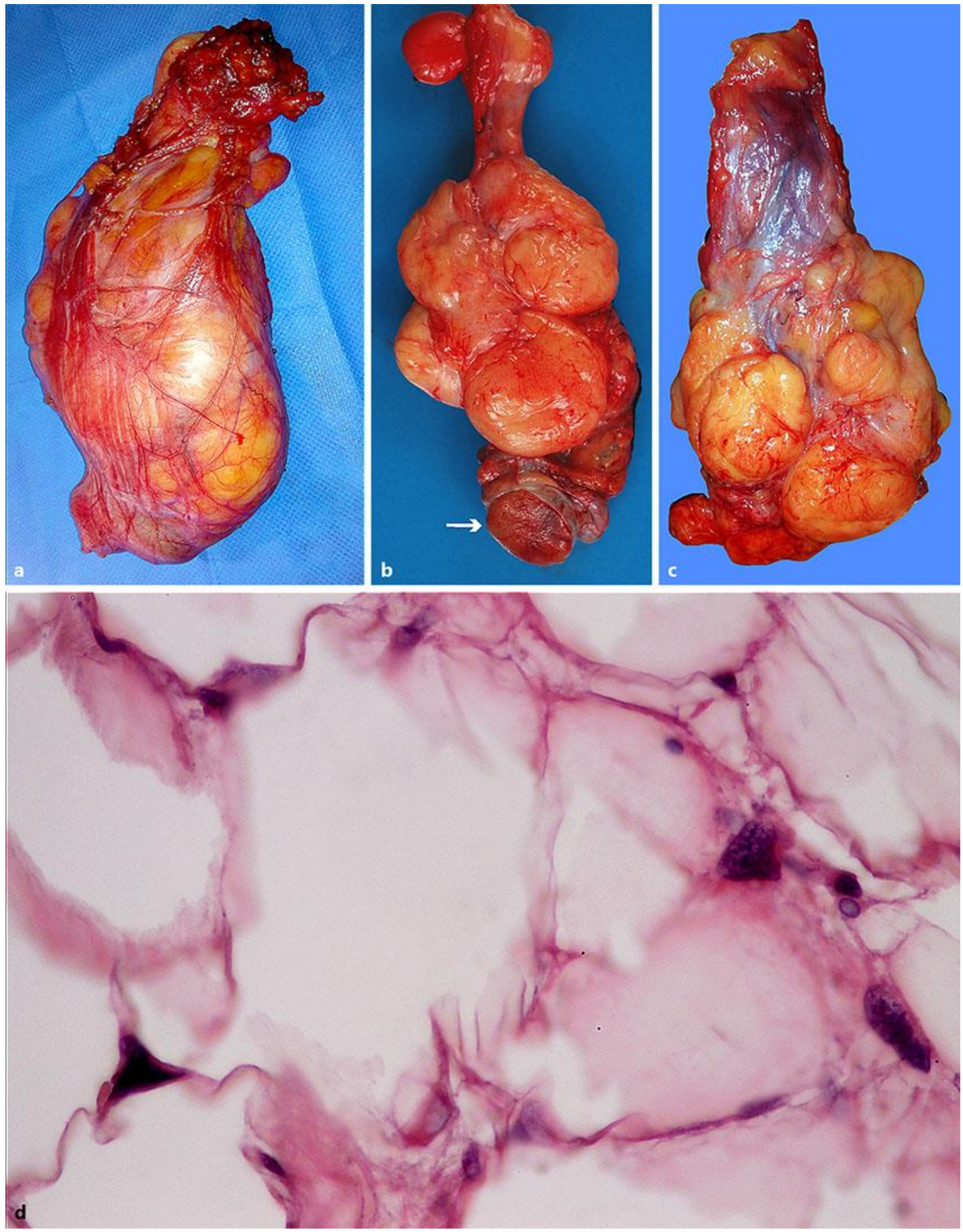

Fig. 2. The spermatic cord was dissected and removed. a It showed a hard lipomatous mass that was $14 \times 8$ $\mathrm{cm}$ in size. The mass involved the entire circumference of the cord and could not be separated from the cord. b The mass had a "bunch of grapes" appearance and consisted of several masses of various sizes that surrounded the spermatic cord. c Macroscopic examination of the testis showed that it appeared to be normal. d Histopathological examination revealed a well-differentiated liposarcoma of the cord with positive margins; some lipoblasts with indented hyperchromatic nuclei were observed (haematoxylin-eosin staining; magnification $\times 400$ ). 